\title{
Superplasticity and Deformation Induced Grain Growth
}

\author{
Eiichi SATO and Kazuhiko KURIBAYASHI
}

The Institute of Space and Astronautical Science, Yoshinodai, Sagamihara, Kanagawa-ken, 229 Japan.

(Received on March 8, 1993; accepted in final form on May 21, 1993)

\begin{abstract}
Superplastic deformation, especially in quasi-stable fine equiaxied grain structures, is accompanied by grain growth whose rate exceeds that which can occur without deformation. The deformation induced component of the grain growth stabilizes the deformation itself through an increase in the flow stress. An empirical expression for the grain growth is demonstrated which describes the behavior of microduplex and second-phase dispersed alloys. Finally, a new deformation model of superplasticity is proposed to explain the grain growth.
\end{abstract}

KEY WORDS: superplasticity; plastic instability; deformation induced grain growth; flow hardening; grain boundary sliding; grain switching.

\section{Introduction}

A material with equiaxed fine grain structure can extremely elongate under a low flow stress when it is deformed at high temperature and under a small strain rate. This is termed "structural superplasticity", and has been known and studied for the last half century. ${ }^{1)}$ Since the 1980's, attempts have been made to utilize superplasticity in materials which are difficult to form such as high strength aluminum alloys and titanium alloys or ceramics. ${ }^{2}$ Originally, superplasticity was observed in alloys whose microstructures remained fine throughout the deformation. Currently, there is some interest in materials which have unstable and unrecrystallized microstructures for their use in practical applications, because it is thought that these materials will also deform superplastically at an extremely high speed. ${ }^{3)}$

However even now, there is no clear understanding of classical superplasticity, which occurs in stable equiaxed fine-grained structures. The characteristics of the superplasticity are known as follows ${ }^{1)}$ : macroscopically, a strain rate sensitivity of flow stress is large-usually larger than 0.3 ; microscopically, the grain boundary sliding, not the dislocation activity, plays an important role. In addition, it is known that grain growth is accelerated by deformation. Figure 1 shows an example of the grain growth behavior in $\mathrm{Al}-5 \% \mathrm{Mg}-0.6 \% \mathrm{Mn}$ alloy with and without deformation.

In the past, grain growth has been considered harmful because it destroys the fine microstructure needed for the superplasticity itself. However, grain growth in super-

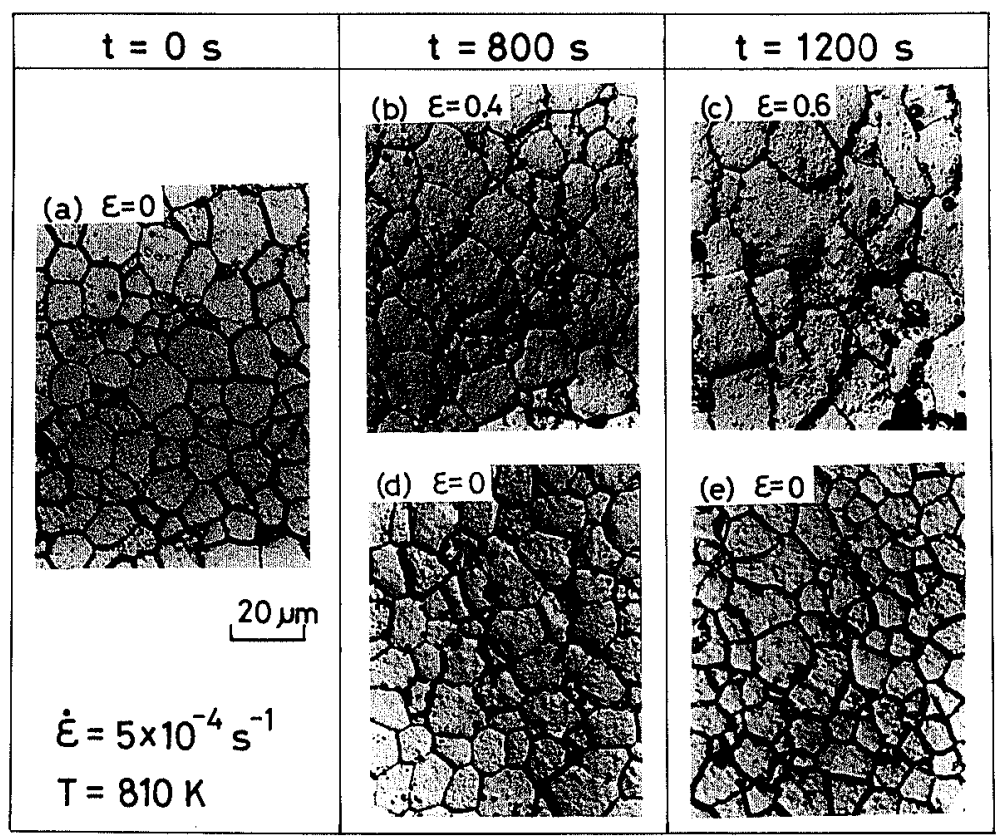

Fig. 1.

Examples of microstructural evolution with and without deformation in $\mathrm{Al}-5 \% \mathrm{Mg}-0.6 \% \mathrm{Mn}$ alloy. ${ }^{4)}$

(a) Before deformation.

(b), (c) Deformed regions where $\varepsilon=0.4$ and 0.6 , respectively.

(d), (e) Undeformed regions in the same conditions with (b) and (c), respectively. 
plasticity is followed by an increase in flow stress, which may stabilize the deformation itself in a similar way to work hardening at ambient temperature. Hardening due to grain growth was not frequently observed because tensile tests were carried out under a constant cross-head speed condition, and not under a constant strain rate condition.

This article describes the grain growth during superplasticity from the viewpoint of its effect on deformation and is based on a recent study by the authors.

\section{The Origin of Large Elongation}

\subsection{Flow Hardening}

Large elongation of superplasticity in tensile deformation is used to being ascribed to a high value of strain rate sensitivity, $m$, in the following manner ${ }^{5,6}$; if one part of a specimen elongates more quickly than the others accidentally, the higher the value of $m$, the more the flow stress in this part increases and the more strongly the subsequent flow in this part is suppressed. Experimentally, a strong correlation between a fracture elongation and $m$-value has been reported. ${ }^{6,7)}$

During superplastic deformation, the flow stress $\sigma$ depends not only on a strain rate $\dot{\varepsilon}$, but also on a grain size $\bar{D}$ and on temperature $T$, but it does not depend on a strain $\varepsilon$ explicitly. Therefore, the flow behavior can be expressed by the modified Dorn's equation: ${ }^{1)}$

$$
\dot{\varepsilon}=\frac{A G b}{k T}\left(\frac{b}{\bar{D}}\right)^{p}\left(\frac{\sigma}{G}\right)^{1 / m} D_{0} \exp \left(\frac{-Q}{R T}\right),
$$

where $D_{0} \exp (-Q / R T)$ is a diffusion constant, $G$ is a shear modulus, $b$ is Burger's vector, $k$ is Boltzman constant, and $A, m$ and $p$ are constants with positive values.

If it is assumed that a grain with an initial size $\bar{D}_{0}$ grows to $\bar{D}$ after a strain $\varepsilon$ according to

$$
\ln \frac{\bar{D}}{\bar{D}_{s}}=\alpha \varepsilon,
$$

it follows that the flow stress changes as

$$
\sigma=K\left(\frac{\bar{D}_{s}}{\bar{D}_{0}}\right)^{m p} \exp (\alpha m p \varepsilon) \dot{\varepsilon}^{m},
$$

where $\bar{D}_{s}$ is a grain size after the annealing of the same time without strain, $\alpha$ is a constant with a positive value, and $K$ includes the terms other than $\bar{D}_{s}, \varepsilon$ and $\dot{\varepsilon}$. The terms $\left(\bar{D}_{s} / \bar{D}_{0}\right)^{m p}$ and $\exp (\alpha m p)$ express the effect of the static and the deformation induced grain growths, respectively.

During a deformation at a constant strain rate, the flow stress increases because of both the static grain growth, which relates to time, and the deformation induced grain growth, which relates to strain (Fig. 2). The former results only in a uniform increase in flow stress, though the latter is expected to stabilize the deformation itself in the form of work hardening at ambient temperature. Consequently, in order to discuss the plastic instability, we must consider the stress increase due to the deformation induced grain growth in addition

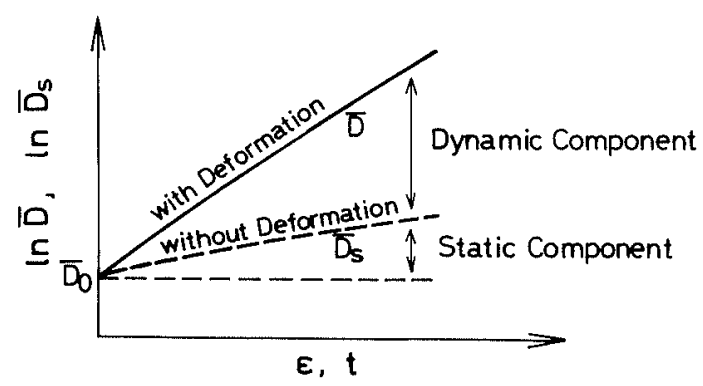

Fig. 2. Schematic drawing of grain growth during superplasticity.

to $m$.

Though Wilkinson et $a l^{8,9)}$ first pointed out the stabilizing effect of grain growth, it was Hamilton who distinguished between the strain induced and static grain growths; he called the former stress increase "flow hardening". ${ }^{10)}$ The parameter $\gamma$ of the flow hardening is defined as

$$
\gamma \equiv \frac{\partial \ln \sigma}{\partial \varepsilon}=\alpha m p
$$

\subsection{Plastic Instability}

In this section we discuss the plastic instability based on the following constitutive equation,

$$
\sigma=K^{\prime} \exp (\gamma \varepsilon) \dot{\varepsilon}^{m},
$$

which considers only the macroscopic dependence of $\sigma$ on $\varepsilon$ and $\dot{\varepsilon}$. In the deformation at ambient temperature, $\sigma$ depends little on $\dot{\varepsilon}$, and the criterion by Considere $^{11)}$ - the deformation is stable while $\gamma$ is larger than 1 -is well known. For the high temperature case, where $\sigma$ depends not only on $\varepsilon$ but also on $\dot{\varepsilon}$, the analysis by Hart $^{12)}$ is widely accepted. This author regarded the stable deformation as one where the difference between cross sections of the uniform and necked regions does not increase, and derived $m+\gamma \geq 1$ as a stable criterion.

However, because the cross section of the specimen decreases as the progress of deformation, we must consider not the difference but the ratio of the cross sections between the uniform and necked regions. The analysis based on the ratio of the cross sections was done by Duncombe, ${ }^{13)}$ who obtained the instability criterion as $\gamma \geq 1$, which is the same as that of Considere. ${ }^{11)}$ Both Hart $^{12)}$ and Duncombe ${ }^{13)}$ analyzed the simplest situation where a neck was formed when it happened to elongate more quickly than the other parts. Thereafter, some detailed analyses were reported, but no essential discussion was added.

In typical superplasticity, $\gamma$, because of grain growth, is not large enough to satisfy Considere's criterion. This means that superplastic deformation proceeds outside of the stable condition. On the other hand, in a specimen extremely elongated, the necked region elongates much more than the rest until it becomes very long and slender.

Considering these two points, the present authors propose the following conditions for good superplasticity:

1) the neck grows broad rather than sharp, and

2) the specimen does not rupture before the necked 
region greatly elongates.

The rupture criterion 2 ) is determined by microstructural behavior like cavitation and it cannot be analyzed by macroscopic parameters such as $m$ and $\gamma$. The broad neck criterion 1) was first pointed out by the authors. ${ }^{18,19)}$ In the following we derive this criterion.

We first consider the cross section ratio to the uniform region $\phi(0<\phi \leq 1)$ at each point of the specimen. Next, the sharpness of a neck at that point is defined by the gradient of $\phi,|\partial \phi / \partial x|$, where $x$ refers to the position in the specimen. The derivative of the gradient of $\phi$ on time $t$ is given by

$$
\frac{\partial}{\partial t}\left|\frac{\partial \phi}{\partial x}\right|=-\dot{\varepsilon}_{u}\left\{(2+I) \phi^{I}-1\right\}\left|\frac{\partial \phi}{\partial x}\right|,
$$

and

$$
I \equiv(\gamma-1) / m,
$$

where $\dot{\varepsilon}_{u}$ is a strain rate of the uniform region. It is calculated by the volume conservation and stress equilibrium conditions under an assumed uniaxial stress distribution in the specimen.

If $\left\{(2+I) \phi^{I}-1\right\}$ is positive, Eq. (6) has a negative value, i.e. a neck broadens. The sign of this value is classified by the value of $I$ : it always has a positive value when $0>I \geq-1$, it always has a negative value when $I \leq-2$, and when $-1>I>-2$, it is negative while $\phi$ is larger than a certain value, and positive after $\phi$ becomes smaller than that value. Incidentally, the derivative of $\phi$ itself is calculated as

$$
\frac{\partial \phi}{\partial t}=\dot{\varepsilon}_{u} \phi\left(1-\phi^{I}\right)
$$

This can easily be used to give Duncombe's criterion: if $I \geq 0$, i.e. if $\gamma \geq 0$, the cross section ratio does not decrease.

Summarizing the above discussion, we can classify the plastic instability as follows (see Table 1):

1) If $I \geq 0$, a neck does not grow; this corresponds to the criterion of $\gamma \geq 0$ given by Duncombe.

Table 1. Summary of plastic instability. ${ }^{18,19)}$

\begin{tabular}{ccc}
\hline$I$ & $m$ and $\gamma$ & \multicolumn{1}{c}{ Behavior of a neck } \\
\hline$I \geq 0$ & $\gamma \geq 1$ & A neck does not grow. \\
$0>I \geq-1$ & $\gamma<1$ & A neck grows but always broadens. \\
& $m+\gamma \geq 1$ & \\
$-1>I>-2$ & $m+\gamma<1$ & A neck sharpens initially but broadens \\
& $2 m+\gamma>1$ & finally. \\
$-2 \geq I$ & $2 m+\gamma \leq 1$ & A neck becomes sharp. \\
\hline
\end{tabular}

2) When $0>I \geq-1$, a neck grows but always broadens. The criterion of $I \geq-1$ corresponds to that of $m+\gamma \geq 1$ given by Hart.

3) When $-1>I>-2$, a neck always grows and initially becomes sharper, but finally becomes broader.

4) In the case where $I \leq-2$, a neck always grows rapidly to become sharper and sharper.

Figure 3 shows some examples of neck evolution with several values of $I$. When $I=-0.5$ or -1.5 , a broad neck is formed and a large elongation may occur. However, when $I=-2.5$, a sharp neck is formed and only a little elongation is obtained.

The above discussion implies that the criterion of superplasticity is given by

$$
I>-2 \text { or } 2 m+\gamma>1 \text {. }
$$

The authors measured $m=0.4$ and $\gamma=0.4$ under a typical superplastic condition in $\mathrm{Zn}-22 \% \mathrm{Al}$ eutectic alloy, ${ }^{20}$ ) and $m=0.6$ and $\gamma=0.8$ in $\mathrm{Al}-33 \% \mathrm{Cu}$ eutectic alloy; ${ }^{21)}$ both satisfy the criterion of Eq. (9). Sometimes the superplastic criterion, $m>0.3$, is used; it is given by Eq. (9) with $\gamma=0.4$

Attempts have been made to predict the fracture strain, a parameter easy to understand and measure. For example, the fracture strain of a material is assumed to be a strain in the uniform region when the cross section of the neck becomes 0 . This can be calculated as a function of $m$ and $\gamma^{16,17,22)}$ In order to discuss the fracture strain, however, we have to know the fracture criterion, which is determined by microscopic behavior such as cavitation. At present the authors feel that it is impossible to estimate a fracture strain.

\section{Grain Growth Behavior}

Some experimental observations showing that grain growth is accelerated by superplastic deformation have been reported for various materials. ${ }^{8-10,23-32)}$ However, except for recent works by the authors, ${ }^{19,20)}$ no one has analyzed in detail the deformation induced component separately from the entire grain growth.

During superplasticity, many kinds of microstructural change may occur depending on the initial microstructure. At first, the so-called "continuous recrystallization" occurs in the deformation of a material which keeps the unrecrystallized microstructure even at high temperature after heavy rolling. ${ }^{33)}$ It is considered that the misorientation of sub-boundaries increases during deformation until sub-boundaries become grain boundaries. During this microstructural change, (sub-) grains grow rapidly, though this must be due to a

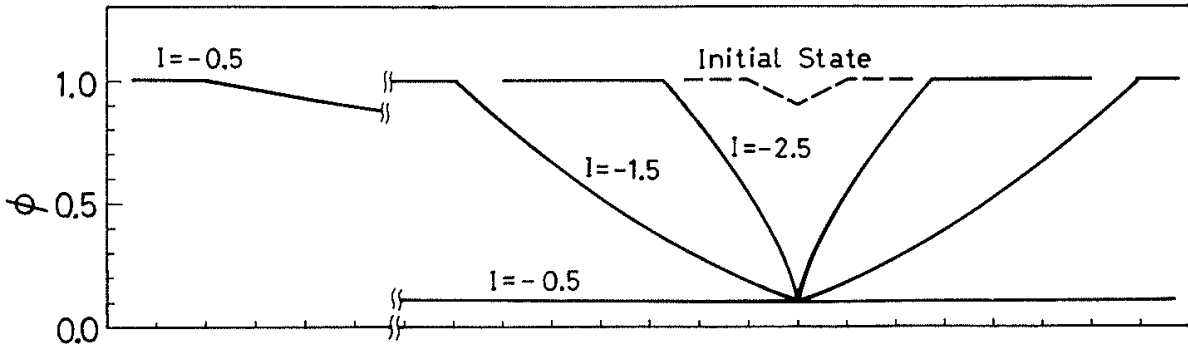

$x$ I arb. unit
Fig. 3.

Examples of neck evolution. A neck indicated by the broken line evolves into the necks indicated by solid lines according to the value of $I .{ }^{18,19)}$ 
different phenomenon than that discussed in this article.

Next, in some classical superplastic alloys, an elongated grain structure may be present from previous extrusion or rolling, but at the beginning of superplastic deformation, the elongated grains quickly become smaller and equiaxied. ${ }^{23-25}$ ) With this type of grain separation, grain growth cannot be discussed qualitatively.

Thirdly, if the microstructure is not fine enough for superplastic deformation at a given temperature and strain rate, lattice dislocations are activated and/or cavities at grain boundaries are formed. In this case, we again cannot discuss grain growth alone.

Finally, even if we obtain a specimen with fine, stable and equiaxed microstructure, we cannot avoid grain growth during deformation at high temperature for long periods. Therefore, it is important to separate the deformation induced component from the entire grain growth.

If we assume that the static and deformation induced grain growths are governed by different mechanisms, then the total rate of grain growth is equal to the sum of their components. However, the entire amount of grain growth cannot be described by the sum of the amounts of the two individual components because their grain size dependencies are different. Clark and Alden analyzed their experimental data assuming that the deformation induced component is approximately given by $\left(\bar{D}-\bar{D}_{s}\right) /$ $\bar{D}_{s}{ }^{28)}$ This estimation does not work when the amount of grain growth is large, and it may result in incorrect description, as described in Sec. 3.2.

The present authors estimated the deformation induced component at $\ln \left(\bar{D} / \bar{D}_{s}\right) \cdot{ }^{19,20)}$ The estimation is accurate within a $15 \%$ error whereas the static and deformation induced grain growths are less than 1.4 and 2.0 times the initial grain size, respectively. The following two equations have been used in the above error estimation: the first is based on the 4th or 3rd power for the static grain growth,

$$
\bar{D}_{s}^{r}-\bar{D}_{0}^{r}=k t, \quad r=4 \text { or } 3,
$$

and the second is an experimental equation obtained from Eq. (13), which will be shown later, for the deformation induced grain growth,

$$
\bar{D}=\bar{D}_{0} \exp (\alpha \varepsilon) \text {. }
$$

Here, $\bar{D}, \bar{D}_{s}$ and $\bar{D}_{0}$ are defined in Sec. 2.1 and all of them can be measured experimentally.

Figure 4 shows the deformation induced component expressed by $\ln \left(\bar{D} / \overline{D_{s}}\right)$ as a function of strain. This is obtained from previously reported data where the specimen has an equiaxed microstructure without any cavities throughout the deformation. ${ }^{4,20,21,27-29,31 \text { ) }}$

\subsection{In Microduplex Alloys}

Figures 4(a) to 4(c) show some results for microduplex alloys, $\mathrm{Zn}-22 \% \mathrm{Al}$ eutectoid alloys, ${ }^{20,31,29)} \mathrm{Al}-33 \% \mathrm{Cu}$ eutectic alloys ${ }^{21)}$ and $\mathrm{Ti}-6 \% \mathrm{Al}-4 \% \mathrm{~V}$ alloy. ${ }^{27)}$ In each alloy in the region where strain rate is lower than the peak in $m$, the deformation induced component depends

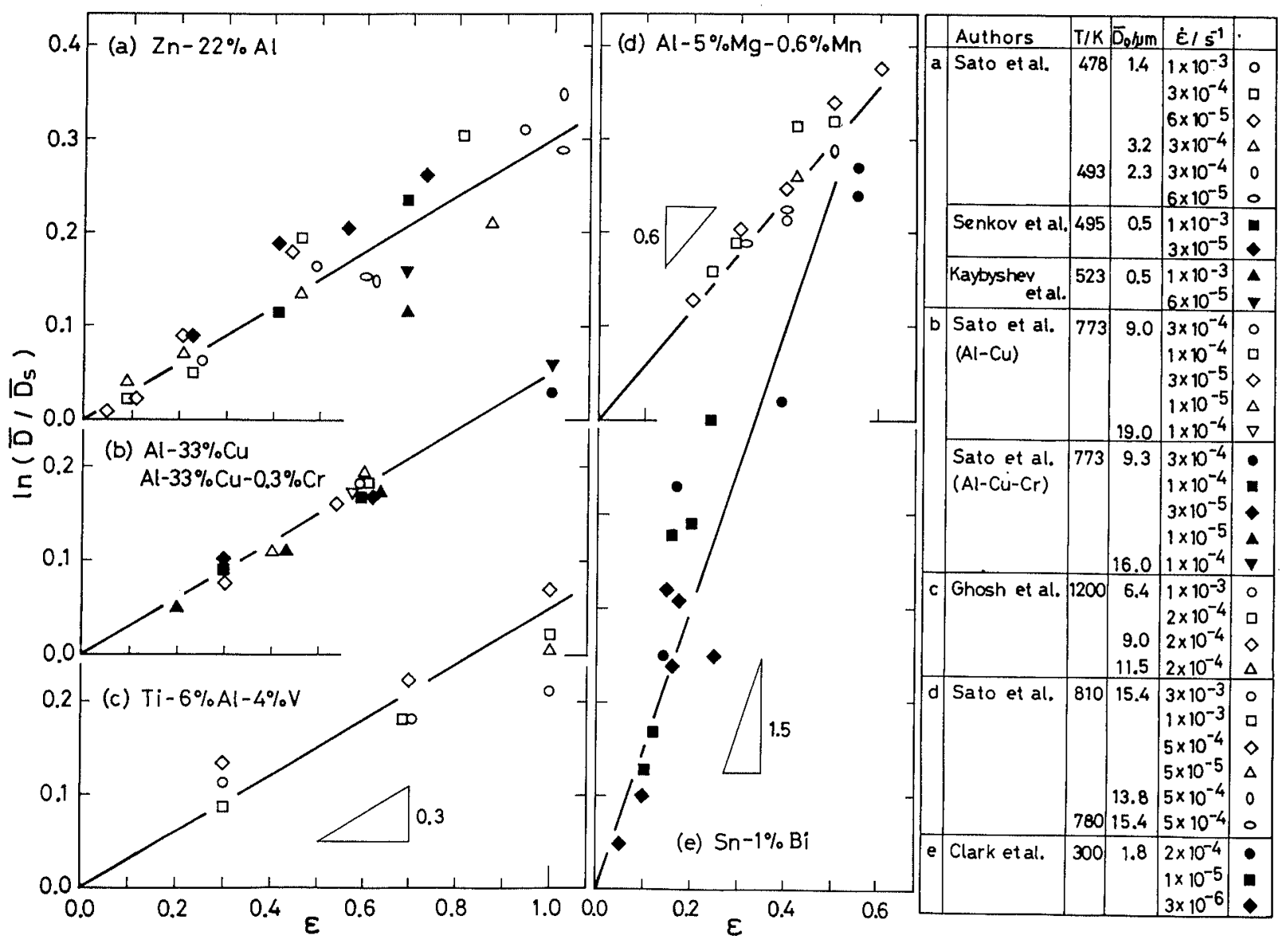

Fig. 4. Summary of previous data on grain growth induced by superplastic deformation in microduplex alloys $(a, b, c),{ }^{20,21,27,29,31)}$ in a second phase dispersed alloy (d) ${ }^{4)}$ and in a single phase alloy (e). ${ }^{28)}$ 
on neither strain rate, temperature nor initial grain size, but is dependent on strain; it is expressed as:

$$
\ln \frac{\bar{D}}{\bar{D}_{s}}\left(\cong \frac{\bar{D}-\bar{D}_{s}}{\bar{D}_{s}}\right)=\alpha \varepsilon,
$$

where the value of $\alpha$ is 0.3 in all alloys.

The left and middle terms of Eq. (12) are almost the same for the case where grain growth is not large. The reason the authors use the logarithmic formula is that it allows the rate of grain growth, derived from the strain and normalized by grain size, to be a constant, $\alpha$, according to the following expression:

$$
\frac{1}{\bar{D}} \frac{\partial \bar{D}}{\partial \varepsilon}=\alpha \text {. }
$$

In contrast, Wilkinson and Càseres reported that the deformation induced component is expressed by Eq. (12) with $\alpha$ being 1.0 in $\mathrm{Cu}$ containing $\mathrm{Zn}-22 \% \mathrm{Al}$ alloy; ${ }^{8}$ it was not measured in pure $\mathrm{Zn}-22 \% \mathrm{Al}$ alloy. The present authors, however, found no difference in grain growth behavior between $\mathrm{Zn}-22 \% \mathrm{Al}-0.5 \% \mathrm{Cu}$ and $\mathrm{Zn}-22 \% \mathrm{Al}$ alloys. ${ }^{19,20)}$ From this experimental result we can conclude that a solute additive does not affect the deformation induced grain growth.

Figure 4(b) shows that the addition of $0.3 \% \mathrm{Cr}$ to $\mathrm{Al}-33 \% \mathrm{Cu}$ alloy, which is more than the solubility limit, causes no difference in the deformation induced grain growth, though it causes some difference in the static grain growth. ${ }^{21)}$ This means that the precipitated third phase also does not affect the deformation induced grain growth.

\subsection{In Second-phase Dispersed Alloy and Single Phase Alloy}

In second-phase dispersed alloy and single phase alloy, it is generally difficult to develop fine grain microstructures because of small pinning forces against grain growth. However, some data can be derived from studies of $\mathrm{Al}-5 \% \mathrm{Mg}-0.3 \% \mathrm{Mn}$ alloy ${ }^{4)}$ (second-phase dispersed structure, as shown in Fig. 4(d)) and of Sn-1\%Bi alloy ${ }^{28)}$ (single phase structure, shown in Fig. 4(e)). The deformation induced component of these materials, expressed by Eq. (12), in the strain rate region below the peak in $m$, is independent of strain rate, temperature and grain size, but proportional to strain. However the value of $\alpha$ is 0.6 in $\mathrm{Al}-\mathrm{Mg}-\mathrm{Mn}$ alloy and $1.5 \mathrm{in} \mathrm{Sn}-\mathrm{Bi}$ alloy, which is much larger than that found in microduplex alloys.

Clark and Alden ${ }^{28)}$ concluded that the deformation induced component calculated by $\left(\bar{D}-\bar{D}_{s}\right) / \bar{D}_{s}$ is at its maximum when the strain rate is at the greatest value of $m$. The data in Fig. 4(e), however, show almost equal deformation induced grain growth for a strain rate that varies over two decades; the data of lower strain rates cannot be used because the static grain growth is too large.

Iwasaki et al. ${ }^{26)}$ reported that the deformation induced grain growth in $7475 \mathrm{Al}$ alloy, whose microstructure has an aspect ratio of 1.8, is expressed by Eq. (12), where $\alpha$ is small, 0.3 .

\subsection{In Ceramics}

The superplastic ceramics can be classified into those which have a large amount of amorphous or liquid phases on grain boundaries and those which have no grain boundary phases. Ceramics with grain boundary phases show little grain growth during deformation, whereas those without show significant grain growth. ${ }^{34)}$ The superplastic behavior and mechanisms in ceramics without boundary phases are expected to be the same as those in alloys.

Highly pure $\mathrm{Al}_{2} \mathrm{O}_{3}$ (alumina) and Y-TZP (Yttria stabilized tetragonal zirconia polycrystal) are regarded as typical of ceramics which do not exhibit large amounts of grain boundary phases. Although $\mathrm{Al}_{2} \mathrm{O}_{3}$ shows rather large amounts of grain growth, ${ }^{46-48)} \mathrm{Y}$-TZP shows rather small amounts. ${ }^{49)}$ Stoto et al. ${ }^{35)}$ observed a grain boundary phase of several nm thickness in Y-TZP which contained a small amount of $\mathrm{SiO}_{2}$; almost every Y-TZP is believed to contain some amount of $\mathrm{SiO}_{2}$.

Yoshizawa and Sakuma ${ }^{32)}$ reported that the deformation induced component of Y-TZP is in proportion to strain but independent of temperature: this is similar to alloys, but the reported value of $\alpha$ is smaller, 0.15 .

\subsection{Summary}

The characteristics of the grain growth induced by superplastic deformation are summarized:

1) it is independent of strain rate, grain size and temperature, and

2) it is proportional to strain, in the region where grain boundary sliding is dominant, i.e. where strain rate is below the peak in $m$. The proportional parameter, $\alpha$, has a value of 0.3 in microduplex alloys, a larger value in second phase dispersed alloys or in single phase alloys, and a smaller value in Y-TZP of ceramics.

\section{Mechanisms of Deformation Induced Grain Growth}

Some models have already been proposed for deformation induced grain growth. ${ }^{28,31,36-40)}$ Unfortunately, the authors must say that no model has been experimentally proven at this juncture.

\subsection{Generation of Excess Vacancies by Grain Boundary Sliding}

Clark and Alden ${ }^{28)}$ and Senkov and Myshlyaev ${ }^{31)}$ proposed that grain boundary sliding produces excess vacancies; those accelerate diffusion and hence grain growth. Though we do not have any established theory for the accommodation process of grain boundary sliding in superplasticity, none of the proposed models ${ }^{41-44)}$ ever predict the accelerated grain growth for reasons which will be subsequently discussed.

First, we have some models which rely on diffusional accommodation. ${ }^{41,44)}$ This is explained as changes in the vacancy concentration at grain boundaries according to the normal stress; this change causes a diffusional mass flow from a compressed region to a depressed region inside the grain. This accommodation, however, activates neither diffusion nor grain growth generally because the 
vacancy concentration on the average among all grain boundaries is not increased.

Secondly, another accommodation model ${ }^{42)}$ indicates that the prohibitor of grain boundary sliding (grain boundary triple point or grain boundary ledge) creates lattice dislocations. These traverse the grain, pile up in front of the opposite grain boundary, and then one by one climb to sinks; the last process determines the rate of grain boundary sliding. Here, if these dislocations cut each other, they produce excess vacancies. During superplasticity, however, very little dislocation activity has been observed in transmission electron microscopy. ${ }^{1)}$ This, in itself, cannot deny the existence of some dislocation activities, but at least it can eliminate the possibility of the presence of tangled dislocations and the previously mentioned "cutting" mechanism.

Finally, a third accommodation model ${ }^{43)}$ regards grain boundary sliding as the motion of grain boundary dislocations. The dislocations which have glided and then piled up at a triple point must, in this instance, climb to sinks. In this model, it is also difficult to produce excess vacancies by dislocation cutting.

\subsection{Coalescence of Grains or Particles by Grain Switching}

Holm et al. ${ }^{36)}$ proposed a grain growth model by grain switching. ${ }^{41)}$ In a second-phase dispersed alloy, two particles at triple points coalesce during one switching event, as shown in Fig. 5(a), and this results in some matrix grain growth. The second phase, however, are

(a)

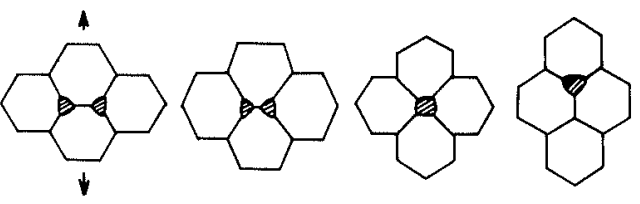

(b)

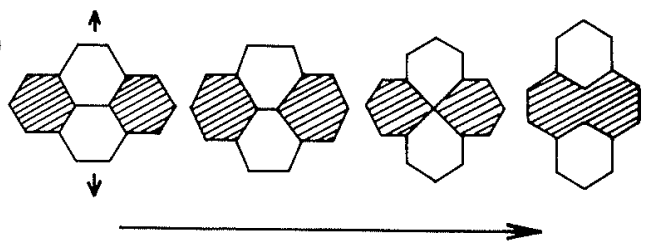

(c)

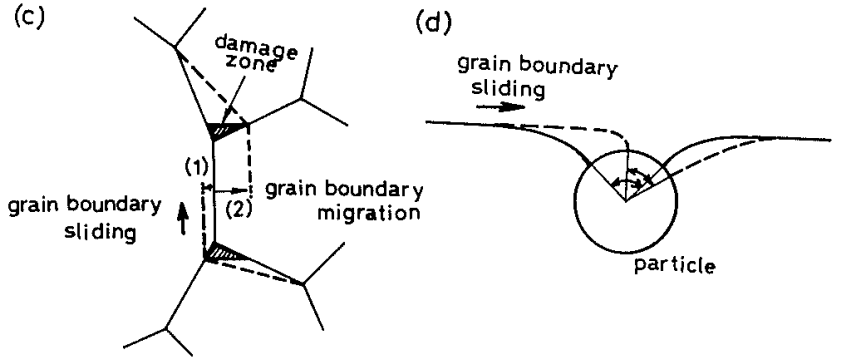

Fig. 5. Previously proposed models of deformation induced grain growth by

(a) particle coalescence by grain switching, ${ }^{36)}$ (b) grain coalescence by grain switching, ${ }^{37)}$ (c) damaged zones at triple points, ${ }^{37)}$ and (d) crimped grain boundary at a particle. ${ }^{38)}$ usually dispersing into many fine particles-unlike Fig. 5(a) where only two particles are situated on one grain boundary-in order to get enough pinning force.

Wilkinson and Càseres ${ }^{37)}$ extended this model into microduplex structures, in which, initially, two separated grains of an identical phase (with hatching) come into contact and coalesce after one switching event, as shown in Fig. 5(b). However, this model cannot explain why the two grains of another phase (white) do not coalesce before being separated.

\subsection{Damaged Zones or Crimped Grain Boundary by Grain Boundary Sliding}

Wilkinson and Càceres ${ }^{37)}$ supposed that two damaged zones are created on the two ends of a sliding grain boundary, and that the grain boundary migrates to consume them in the direction of the larger grain to the smaller grain, as shown in Fig. 5(c). However, the discussion in Sec. 4.1 already excludes the possibility of the existence of tangled dislocations and "damaged" zones.

Furthermore, Wilkinson ${ }^{38)}$ proposed that a sliding grain boundary is crimped by a second phase particle, as shown in Fig. 5(d); this crimping decreases the pinning force of the particle. However, if grain boundary sliding is well accommodated, the grain boundary may remain straight. Any of the above suppositions may be correct, but they cannot be proven without sufficient experimental support.

\subsection{Grain Growth Induced by Grain Switching at an Irregularity}

The present authors re-examined grain switching in an irregular grain morphology which was proposed by Morral and Ashby, ${ }^{44)}$ and the following deformation and grain growth model is proposed. ${ }^{39,40)}$

In two dimensions, as shown in Fig. 6, a pentagon-heptagon pair (5-7 pair) in a quasi-regular hexagonal array of grains can be considered as an edge dislocation in a grain lattice. When a tensile stress is applied oblique to the 5-7 pair, a switching event occurs among $A, B, C$ and $D$ grains $(a \rightarrow b)$. This event can be described as the movement of a 5-7 pair, i.e. the glide motion of an edge dislocation. On the other hand, when a stress is applied parallel to the 5-7 pair, a switching event occurs among $\mathrm{A}, \mathrm{B}, \mathrm{C}$ and $\mathrm{E}$ grains $(\mathrm{c} \rightarrow \mathrm{g})$. This causes the pentagonal grain $A$ to change into $a$ quadrilateral shape (d), to be consumed by its neighbors (e) and then to disappear (f). These events can be described as the climb motion of an edge dislocation.

If we suppose the configurations around the 5-7 pair before and after its movement are identical in shape but not necessarily in size, the glide motion yields some amount of strain, and the climb motion yields strain as well as disappearance of one grain, i.e. grain growth. Supposing the deformation proceeds only by grain switching, we can expect the amount of grain growth is in proportion to the number of the climb motion and also to the strain. The above model of grain growth is similar to that for static grain growth proposed by Hillart $^{50)}$ except that the first step of grain growth (Fig. 
(a)
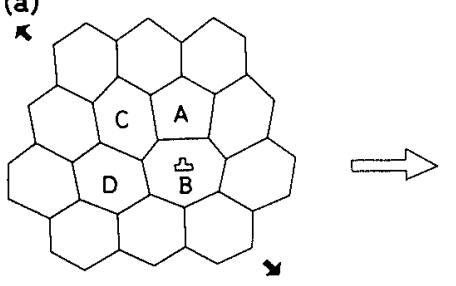

(b)

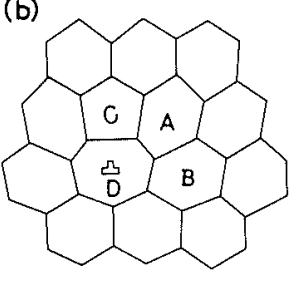

(c)
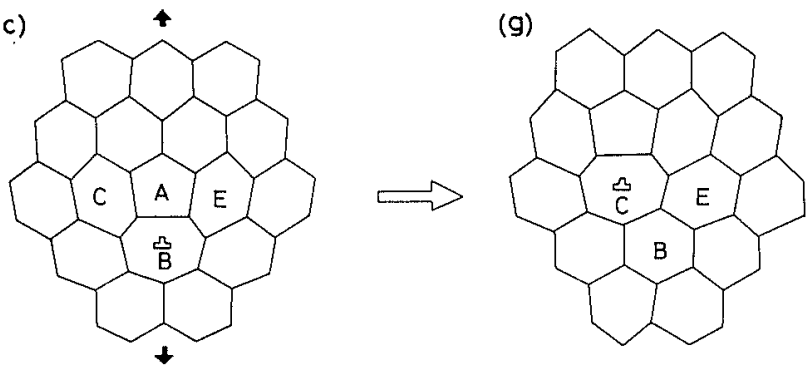

$\sqrt{3}$

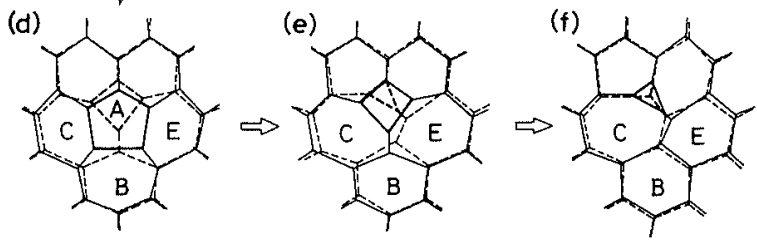

Fig. 6. A model of deformation induced grain growth by grain switching at an irregularity. ${ }^{39,40)}$

(a, b) Glide motion of a 5-7 pair, which yields strain only. (c-g) Climb motion of a 5-7 pair, which yields grain growth and strain.

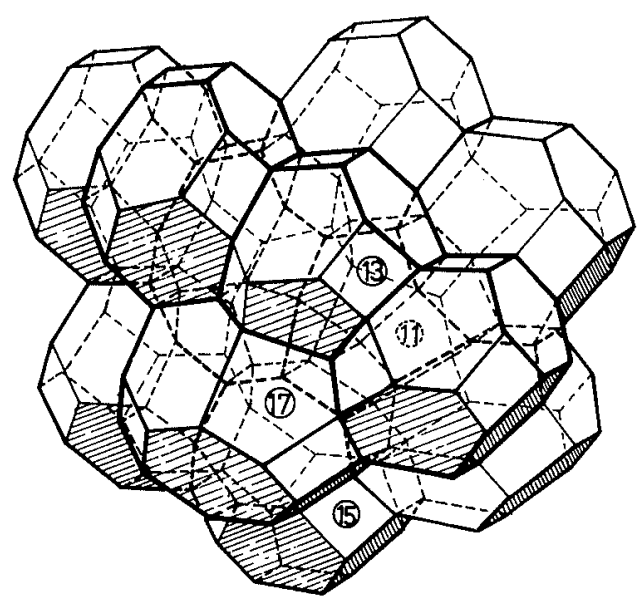

Fig. 7. Three dimensional grain lattice with an irregularity, i.e., an edge dislocation. ${ }^{39,40)}$

6(d)) is assisted by deformation.

Switching that is considered as a movement of an edge dislocation in a grain lattice, can easily be expanded into three dimensions. Figure 7 shows an edge dislocation in a grain lattice where Kelvin's tetra-kai-dekahedra stack in a bcc lattice; the grain at the top of a "half grain plane" is a 17- or 15-faced grain, and the grain next to it is a 13- or 11-faced grain, respectively. These 17-15 and 13-11 pairs coincide with a 5-7 pair in two dimensions. Consequently, we can discuss grain switching in three dimensions in almost the same manner as in two dimensions.
This three dimensional model predicts a rate equation of grain growth exactly the same as that observed, Eq. (13). The value of $\alpha$ is predicted from 0.2 to 0.3 according to several assumed parameters. This value agrees quite well with the experimental value in microduplex alloys.

\section{Conclusions}

Superplastic deformation, especially in quasi-stable fine equiaxed grain structures, is accompanied by grain growth, whose rate exceeds that which occurs without deformation. The deformation induced component of the grain growth stabilizes the deformation itself, through an increase in the flow stress. Analyzing the neck growth behavior results in a broad neck criterion, $(m+\gamma) / m>2$, for a good superplastic deformation.

The characteristics of this deformation induced component have been empirically found to be

1) independent of strain rate, grain size or temperature, and

2) proportional to strain,

in the region where grain boundary sliding is dominant, i.e. strain rate is below the peak in $m$.

A new deformation model of superplasticity is proposed in order to explain the grain growth. A pentagon-heptagon pair is introduced into a hexagonal grain lattice in two dimensions. A grain switching in this lattice can be considered as the movement of an edge dislocation. When this dislocation climbs, grain growth occurs that is proportional only to strain.

\section{REFERENCES}

1) For ex.; J. W. Edington, K. N. Melton and C. P. Cutler: Prog. Mater. Sci., 21 (1976), 61; K. A. Padmanabham and G. J. Davies: Superplasticity, Springer-Verlag, Berlin, (1980).

2) For ex.; Int. Conf. on Superplasticity, Grenoble, (1985); Int. Symp. on Superplasticity in Aerospace, Phoenix, (1988); MRS Int. Meeting on Advanced Materials, Superplasticity, Tokyo, (1988); Int. Conf. on Superplasticity and Superplastic Forming, Blaine, (1988).

3) For ex.; Int. Symp. on Superplasticity in Aerospace II, Anaheim, (1990); MRS Spring Meeting, Superplasticity in Metals, Ceramics and Intermetallics, San Francisco, (1990); Int. Conf. on Superplasticity in Advanced Materials, Osaka, (1991).

4) E. Sato, K. Itaya, K. Kuribayashi and R. Horiuchi: J. Jpn. Inst. Light Met., 39 (1989), 437.

5) W. A. Backofen, I. R. Turner and D. H. Avery: Trans. ASM, $\mathbf{5 7}$ (1964), 980

6) D. H. Avery and W. A. Backofen: Trans. ASM, 58 (1965), 551

7) D. A. Woodford: Trans. ASM, 62 (1969), 291.

8) D. S. Wilkinson and C. H. Càseres: J. Mater. Sci. Lett., 3 (1984), 395.

9) C. H. Càceres and D. S. Wilkinson: Acta Metall., 32 (1984), 415

10) C. H. Hamilton: Strength of Metals and Alloys, ed. by H. J. McQueen and J. P. Bailon, Pergamon, New York, (1986), 1831.

11) C. H. Considere: Ann. Ponts et Chaussees, 9 (1985), 574.

12) E. W. Hart: Acta Metall., 15 (1967), 351.

13) E. Duncombe: Int. J. Mech. Sci., 14 (1972), 325.

14) J. J. Jonas, R. A. Holt and C. E. Coleman: Acta Metall., 24 (1976), 911.

15) M. A. Fortes: Int. J. Mech. Sci., 19 (1977), 483.

16) J. W. Hutchinson and K. W. Neale: Acta Metall, 25 (1977), 839.

17) F. A. Nichols: Acta Metall., 28 (1980), 663.

18) E. Sato, K. Kuribayashi and R. Horiuchi: J. Jpn. Inst. Met., 52 (1988), 1051.

19) E. Sato, K. Kuribayashi and R. Horiuchi: Proc. MRS Int. Meeting on Advanced Materials Vol. 7, ed. by K. Kobayashi and F. Wakai, 
MRS, Pittsburgh, (1989), 165.

$20)$ E. Sato, K. Kuribayashi and R. Horiuchi: J. Jpn. Int. Met., 52 (1988), 1043

21) E. Sato, K. Kuribayashi and R. Horiuchi: J. Jpn. Int. Met., 53 (1989), 885 .

22) A. K. Ghosh: Acta Metall., 25 (1977), 1413.

23) B. M. Watts and M. J. Stowell: J. Mater. Sci., 6 (1971), 228.

24) G. Herriot, M. Suery and B. Baudelet: Scr. Metall., 6(1972), 657.

25) M. Suéry and B. Baudelet: Rev. Phys. Appl., 13 (1978), 53.

26) H. Iwasaki, Y. Irie, S. Hayami, K. Higashi and T. Ito: J. Jpn. Inst. Light Met., 39 (1989), 798.

27) A. K. Ghosh and C. H. Hamilton: Metall. Trans., 10A (1979), 699.

28) M. A. Clark and T. H. Alden: Acta Metall., 21 (1973), 1195.

29) O. A. Kaybyshev, I. V. Kazachkov and V. M. Rozenberg: Fiz. Met. Metalloved, 36 (1973), 1235; trans.: Phys. Met. Metall., 36 (1973), No. 6, 103.

30) F. A. Mohamed, M. M. Ahmed and T. G. Langdon: Metall. Trans, 8A (1977), 933

31) O. N. Senkov and M. M. Myshlyaev: Acta Metall., 34 (1986), 97.

32) Y. Yoshizawa and T. Sakuma: Superplasticity in Advanced Materials, ed. by S. Hori, M. Tokizane and N. Furushiro, The Japan Soc. for Research on Superplasticity, (1991), 251.

33) E. Ness: Superplasticity, ed. by B. Baudelet and M. Suery, CNRS, Paris, (1985), 7-1.

34) R. Raj: Superplasticity and Superplastic Forming, ed. by C. H. Hamilton and N. E. Paton, TMS, Warrendale, (1988), 583.
35) T. Stoto, M. Nauer and C. Carry: J. Am. Ceramic Soc., 74 (1991), 2615.

36) K. Holm, J. D. Embury and G. R. Purdy: Acta Metall., 25 (1977), 1191.

37) D. S. Wilkinson and C. H. Càseres: Acta Metall., 32 (1984), 1335.

38) D. S. Wilkinson: Superplasticity and Superplastic Forming, ed. by C. H. Hamilton and N. E. Paton, TMS, Warrendale, (1988), 81.

39) E. Sato, K. Kuribayashi and R. Horiuchi: MRS Symp. Proc. Vol. 196, ed. by M. J. Mayo, J. Wadsworth, A. K. Mukherjee and M. Kobayashi, MRS, Pittsburgh, (1990), 27.

$40)$ E. Sato, K. Kuribayashi and R. Horiuchi: J. Jpn. Inst. Met., 55 (1991), 839.

41) M. F. Ashby and R. A. Verrall: Acta Metall., 21 (1973), 149.

42) A. Ball and M. M. Hutchison: Met., Sci. J., 3 (1969), 1

43) R. C. Gifkins: Metall. Trans., 7A (1976), 1225

44) J. E. Morral and M. F. Ashby: Acta Metall., 22 (1974), 567.

45) R. Raj and M. F. Ashby: Metall. Trans., 2 (1971), 1113.

46) J. D. Fridez, C. Carry and A. Mocellin: Advances Ceram., 10 (1985), 720.

47) L. A. Xue and I. W. Chen: J. Am. Ceramic Soc., 73(1990), 3518

48) J. Besson and M. Abouaf: Acta Metall. Mater., 39 (1991), 2225.

49) T. G. Nieh and J. Wadsworth: J. Am. Ceramic Soc., 72 (1989), 1469.

50) M. Hillart: Acta Metall., 13 (1965), 227.

(Originally published in Tetsu-to-Hagané, 78 (1992), 1414, in Japanese) 